\title{
Changing clinical patterns in rheumatoid arthritis management over two decades: sequential observational studies
}

\author{
Aneela N Mian ${ }^{1,2^{*}}$, Fowzia Ibrahim², Ian C Scott ${ }^{2}$, Sardar Bahadur ${ }^{3}$, Maria Filkova ${ }^{2}$, Louise Pollard ${ }^{3}$, Sophia Steer ${ }^{1,2}$,
} Gabrielle H Kingsley, ${ }^{2,3}$, David L Scott ${ }^{1,2}$ and James Galloway ${ }^{1,2}$

\begin{abstract}
Background: Rheumatoid arthritis (RA) treatment paradigms have shifted over the last two decades. There has been increasing emphasis on combination disease modifying anti-rheumatic drug (DMARD) therapy, newer biologic therapies have become available and there is a greater focus on achieving remission. We have evaluated the impact of treatment changes on disease activity scores for 28 joints (DAS28) and disability measured by the health assessment questionnaire scores (HAQ).

Methods: Four cross-sectional surveys between 1996 and 2014 in two adjacent secondary care rheumatology departments in London evaluated changes in drug therapy, DAS28 and its component parts and HAQ scores (in three surveys). Descriptive statistics used means and standard deviations (SD) or medians and interquartile ranges (IQR) to summarise changes. Spearman's correlations assessed relationships between assessments.

Results: 1324 patients were studied. Gender ratios, age and mean disease duration were similar across all cohorts. There were temporal increases in the use of any DMARDs (rising from $61 \%$ to $87 \%$ of patients from 1996-2014), combination DMARDs (1 \% to $41 \%$ ) and biologic (0 to $32 \%$ ). Mean DAS28 fell (5.2 to 3.7), active disease (DAS28 > 5.1 ) declined (50 \% to $18 \%$ ) and DAS28 remission (DAS28<2.6) increased (8 \% to $28 \%$ ). In contrast HAQ scores were unchanged (1.30 to 1.32) and correlations between DAS28 and HAQ weakened (Spearman's rho fell from 0.56 to 0.44$)$.

Conclusions: Treatment intensity has increased over time, disease activity has fallen and there are more remissions. However, these improvements in controlling synovitis have not resulted in comparable reductions in disability measured by HAQ. As a consequence the relationship between DAS28 and HAQ has become weaker over time. Although the reasons for this divergence between disease activity and disability are uncertain, focussing treatment entirely in suppressing synovitis may be insufficient.
\end{abstract}

Keywords: Rheumatoid arthritis, Disease activity, Disability, Epidemiology, Clinical outcomes

\section{Background}

Rheumatoid arthritis (RA) management paradigms have changed over the last two decades. There is now greater emphasis on intensive suppressive treatment with greater use of disease modifying anti-rheumatic drugs

\footnotetext{
* Correspondence: aneela.mian@nhs.net

'Department of Rheumatology, King's College Hospital, Denmark Hill, London SE5 9RS, UK

2Department of Rheumatology, King's College School of Medicine, King's College London, Weston Education Centre, 10 Cutcombe Road, Denmark Hill, London SE5 9RJ, UK

Full list of author information is available at the end of the article
}

(DMARDs), including combinations of DMARDs, and the use of biologic treatments when standard DMARDs are insufficient [1]. The impact of treatment can be assessed using standard methods such as the Disease Activity Score for 28 joints (DAS28) [2] and the Health Assessment Questionnaire Disability Index (HAQ) [3]. In recent years 'Treat to Target' has provided a management framework. It emphasises that the treatment goal should be achieving remission [4]. Several groups have shown that the frequency of severe disease is declining and there are growing numbers of patients with 
moderate or low DAS28 scores [5-8]. Despite these findings, studies from the Netherlands have suggested that although disease activity levels have improved over time, levels of disability may actually be increasing [9]. We consider further information is needed about the changing patterns of DAS28 and HAQ scores in RA patients seen in routine treatment settings over the last 20 years. We have undertaken such an evaluation in two adjacent English rheumatology units over two decades examining the changes in treatment, disease activity and disability levels over time in sequential cross-sectional studies.

\section{Methods}

\section{Setting}

Cross-sectional data were collected at four time points from secondary care rheumatology departments at King's College Hospital and University Hospital Lewisham; these are two adjacent centres in South London. The data were collected prospectively by different trained clinicians using standardised clinical assessments in: (i) 1996-97; (ii) 2001-3; (iii) 2009-10; (iv) 2013-14. The surveys assessed consecutive consenting outpatients attending specialist rheumatology clinics within routine clinical care settings.

\section{Patients}

The hospitals serve ethnically diverse inner city populations with broad ranges of social and financial status. Patients included had a consultant diagnosis of RA by the 1987 criteria of the American College of Rheumatology. Disease duration was calculated from date of rheumatologist diagnosis.

Data for the first three cohorts were collected as surveys undertaken as part of routine care evaluations; these have been previously published [10-12]. The patients were consecutive outpatient attenders; there were no exclusion criteria. Only patients in whom full data for DAS28 components had been collected were studied; this meant excluding $3 \%$ of the previously reported patients. Data for the fourth cohort were extracted from routinely captured clinic attendance electronic patient records; full data was available during the survey period in 520 RA patients from 1200 attenders with inflammatory arthritis.

\section{Assessments}

The data collected comprised demographic details (age, sex and disease duration), information about disease activity including the disease activity score for 28 joints (DAS28) and its component parts with ESR, and the use of synthetic DMARDs, biologic DMARDs and oral steroids. We also collected information about disability scores using the Health Assessment Questionnaire
(HAQ), pain (100 mm visual analogue scores) and fatigue (100 mm visual analogue scores) completed by the patients were collected in the first, second and fourth cohorts (for pain) and second and fourth cohort (for fatigue). HAQ scores, pain VAS and fatigue measures were not collected in the third cohort. Patients were assessed prospectively by medical and nursing staff who had been trained in RA assessments.

\section{Analysis}

Statistical analysis used STATA statistical software (version 13). Age, disease duration, disease activity assessments and other outcomes were described using means and standard deviations (SD) or medians and interquartile ranges (IQR) for non-normal data. DAS28 category proportions were given as raw figures and percentages. DAS28 remission was defined as a score of $<2.6$, low disease activity $2.6-3.2$, moderate disease activity $3.2-5.1$, sever disease $>5.1$. Spearman's correlations were used to assess relationship between the DAS28 and HAQ.

\section{Local approvals}

Collecting data for the first cohort received approval from King's College Hospital Local Research Ethics Committee; patients provided written informed consent. The third and fourth cohorts utilised data collected through departmental audits. Approval for these collections was obtained from the Trust audit division. All data was anonymised before analysis. No additional data were collected from patients for the purpose of the current study. As the analysis was the secondary use of existing anonymised data our local research and development office approved its use in line with current national research governance arrangements.

\section{Results}

The four cohorts of RA patients seen between 1996 and 2014 ranged in size from 189 to 520 patients. Overall 1324 patients were studied. The groups had similar demographic features (Table 1): 76-80 \% were female; their mean ages were 58-60 years; and their mean disease durations were 9.1-10 years.

Over time more patients received suppressive therapy: in 1996-97 only $61 \%$ of patients received one or more DMARDs; by 2013-14 $87 \%$ of patients were receiving them (Table 1). The intensity of suppressive treatment increased over time, with rising numbers of patients receiving combination DMARDs and biologics. Combination DMARD use was $1 \%$ in 1996-97 and increased to $41 \%$ by 2013-14. No biologics were available in 1996-97; their use increased from $4 \%$ in 2001-03, $17 \%$ in 2009-10 and further still to $32 \%$ in 2013-14. In contrast, systemic steroid use was unchanged. 
Table 1 Characteristics of patient cohorts

\begin{tabular}{|c|c|c|c|c|}
\hline \multirow[t]{2}{*}{ Characteristic } & 1996-97 & $2001-03$ & $2009-10$ & $2012-14$ \\
\hline & $n=189$ & $n=310$ & $n=304$ & $n=520^{*}$ \\
\hline Female gender, n (\%) & $140(74 \%)$ & $237(76 \%)$ & $244(80 \%)$ & $413(79 \%)$ \\
\hline Age, mean (SD) & $59(14)$ & $60(13)$ & $59(15)$ & $58(15)$ \\
\hline Disease duration in years, median (IQR) & $8(13)$ & $9(10)$ & $10(9)$ & $10(9)$ \\
\hline Any DMARD, n (\%) & $115(61 \%)$ & $199(64 \%)$ & $242(80 \%)$ & $420(87 \%)$ \\
\hline Combination DMARDs, n (\%) & $1(1 \%)$ & $31(10 \%)$ & $65(21 \%)$ & 199 (41\%) \\
\hline Biologic users, n (\%) & 0 & $11(4 \%)$ & $53(17 \%)$ & $155(32 \%)$ \\
\hline Oral steroids, n (\%) & $23(12 \%)$ & $70(23 \%)$ & $30(10 \%)$ & $56(12 \%)$ \\
\hline DAS, mean (SD) & $5.2(1.6)$ & $4.7(1.6)$ & $3.8(1.5)$ & $3.7(1.6)$ \\
\hline Tender joint count, mean (SD) & $10.1(9.2)$ & $7.5(7.3)$ & $4.1(5.6)$ & $4.2(6.2)$ \\
\hline Swollen joint count, mean (SD) & $7.1(5.5)$ & $4.8(4.1)$ & $2.9(4.2)$ & $1.5(2.7)$ \\
\hline Patient global, mean (SD) & $50(25)$ & $50(29)$ & $40(25)$ & $35(28)$ \\
\hline ESR, mean (SD) & $35(26)$ & $33(27)$ & $26(21)$ & $19(21)$ \\
\hline HAQ score, median (IQR) & $1.30(1.52)$ & $1.52(0.79)$ & Not available & $1.32(0.84)$ \\
\hline Pain, Mean (SD) & $50(24)$ & $48(28)$ & Not available & $44(28)$ \\
\hline Fatigue, Mean (SD) & Not available & $50(28)$ & Not available & $51(26)$ \\
\hline
\end{tabular}

Legend: $S D$ standard deviation, IQR interquartile range, ${ }^{*} n=484$ for DMARD data

Disease activity fell over time, with the proportion of patients in remission increasing from $8 \%$ in the earliest cohort to $28 \%$ in the most recent (Table 2). In parallel, the proportion with severe disease fell from $50 \%$ in the earliest cohort to $18 \%$ in the most recent, whilst the proportion with moderate disease remained constant. There are differences in the relative contribution of the individual components of DAS28 across disease activity strata (Table 2). Most noticeable are the reductions in swollen joint counts in all strata compared with the increases in patient global scores in most strata. The mean pain VAS improved with each consecutive cohort from 50 in 1996-97, 48 in 2001-03, and 44 in 2013-2014 but mean fatigue scores did not fall (see table).

In contrast to measures of disease activity, there was no evidence of a fall in disability levels over time. Mean HAQ scores were 1.30 in 1996-97 and 1.32 in 2013-14, with a slightly higher mean HAQ of 1.52 in 2001-03. Comparing disability change over time across the disease activity groups, mean HAQ scores in patients in remission and low disease activity states were higher in 2013-14 compared to 1996-97 (Table 2). Associated with this finding the overall correlation between disease activity and disability became weaker over time; in 1996-97 Spearman's rho was 0.56 , in 2001-03 it was 0.55 and in 2013-14 it was 0.44 .

\section{Discussion}

Our consecutive cross-sectional surveys across two collaborating adjacent specialist units in London between 1996 and 2014 showed the changing pattern of RA treatment and disease severity. The key changes were: increases in the use of DMARDs, combination DMARDs and biologics; falls in mean DAS28; and increases in DAS28 remissions. However, HAQ scores were unchanged and correlations between DAS28 and HAQ weakened. The age, gender and disease duration of the four cohorts were similar and consequently changes in DAS28 could not be explained by differences in demographic factors.

There are several possible explanations for the decline in disease severity: RA may be becoming a milder disease; milder cases of RA may be attending outpatient clinics; or RA treatments are becoming more effective. We noted a minor non-significant difference in disease durations in the last two cohorts compared with earlier cohorts (increasing from 8 years to 10 years). We cannot be certain that this did not have some impact on disability levels but it is unlikely to have been substantial.

Our findings suggest a trend towards milder RA. This has been highlighted in studies of inception cohorts of RA patients [13-16]. Similar reduction in disease activity levels have also been described in established RA, and these reductions preceded the introduction of biologic agents [6]. There are several possible explanations for these observed declines in disease severity. Firstly RA may be becoming a milder disease. Secondly, milder cases of RA may be attending outpatient clinics. Finally RA treatments may be becoming more effective. A North American analysis of patients seen over three consecutive decades from 1970 suggested the reduction in disease severity could be explained entirely by adjusting for DMARD exposure, indicating increased treatment accounts for the improvement [8]. Irrespective of why 
Table 2 Comparison of DAS28 components and disability status across disease activity strata

\begin{tabular}{|c|c|c|c|c|}
\hline DAS28 Group & $<2.6$ & $2.6-3.2$ & $3.2-5.1$ & $>5.1$ \\
\hline \multicolumn{5}{|l|}{ 1996-97 Cohort } \\
\hline Number of patients, (\%) & $15(8 \%)$ & $8(4 \%)$ & $72(38 \%)$ & $94(50 \%)$ \\
\hline Tender joint count, mean (SD) & $0.7(1.4)$ & $0.8(1.4)$ & $4.9(4.8)$ & $16.4(8.5)$ \\
\hline Swollen joint count, mean (SD) & $0.3(0.9)$ & $4.0(3.6)$ & $4.5(3.3)$ & $10.5(5.1)$ \\
\hline Patient global, mean (SD) & $14(7)$ & $26(16)$ & $42(20)$ & $64(21)$ \\
\hline ESR, mean (SD) & $12(7)$ & $15(8)$ & $30(27)$ & $44(25)$ \\
\hline $\mathrm{HAQ}$, mean (SD) & $0.3(0.4)$ & $0.8(0.8)$ & $1.0(0.8)$ & $1.7(0.8)$ \\
\hline \multicolumn{5}{|l|}{ 2001-03 Cohort } \\
\hline Number of patients, (\%) & $29(9 \%)$ & $32(10 \%)$ & $115(37 \%)$ & $135(43 \%)$ \\
\hline Tender joint count, mean (SD) & $0.4(0.7)$ & $1.3(1.6)$ & $4.6(4.2)$ & $12.9(7.1)$ \\
\hline Swollen joint count, mean (SD) & $0.8(1.4)$ & $2.3(2.6)$ & $3.5(2.8)$ & $7.5(4.0)$ \\
\hline Patient global, mean (SD) & $15(19)$ & $28(22)$ & $42(22)$ & $70(22)$ \\
\hline ESR, mean (SD) & $9(7)$ & $20(19)$ & $26(21)$ & $48(28)$ \\
\hline $\mathrm{HAQ}$, mean (SD) & $0.7(0.7)$ & $1.0(0.7)$ & $1.4(0.7)$ & $1.9(0.6)$ \\
\hline \multicolumn{5}{|l|}{ 2013-14 Cohort } \\
\hline Number of patients, (\%) & $148(28 \%)$ & $67(13 \%)$ & 212 (41%) & $93(18 \%)$ \\
\hline Tender joint count, mean (SD) & $0.7(1.6)$ & $1.6(2.4)$ & $4.7(4.7)$ & $15.2(7.9)$ \\
\hline Swollen joint count, mean (SD) & $0.2(0.7)$ & $1.0(1.6)$ & $1.9(2.3)$ & $5.3(3.6)$ \\
\hline Patient global, mean (SD) & $24(17)$ & $24(17)$ & $50(21)$ & $73(16)$ \\
\hline ESR, mean (SD) & $9(7)$ & $22(17)$ & $26(18)$ & $34(23)$ \\
\hline $\mathrm{HAQ}$, mean (SD) & $0.9(0.8)$ & $1.2(0.8)$ & $1.4(0.8)$ & $1.9(0.6)$ \\
\hline
\end{tabular}

Legend: $S D$ standard deviation

fewer patients have highly active RA, its impact is reflected by declining rates of hand and foot surgery for RA [17].

Studying the DAS28 strata in the different cohorts showed differences over time between subjective and objective components of DAS28. Swollen joint counts fell but patient global responses did not. Composite measures like DAS28 are influenced by many factors in addition to the inflammatory burden of RA. These other factors include comorbidity, especially psychological problems such as depression, and other features of the disease such as pain [18]. The fact that swollen joint counts have fallen so substantially across all disease activity strata implies that the control of synovitis by modern treatment is more effective than would appear to be the case from DAS28 scores alone. The most prevalent RA state under follow up in contemporary practice is moderately active disease, which accounted for $41 \%$ of the most recent cohort, and an evidencebased management strategy is needed for this group.

Throughout the time of the four cross sectional surveys, there was greater emphasis on the use of DMARDs, DMARD combinations and biologics. There has also been an increase in the involvement of specialist nurses. We consider these developments are the most likely explanation of these changes. The greater emphasis on seeing
RA patients early may also have influenced the findings. However, the introduction of formal early arthritis clinics in both centres did not occur until after the last survey was completed.

The relationship between disease activity and disability has been studied in detail in historic cohorts, where it was apparent that disease activity was the key driver of disability in early disease, whilst erosive damage became more important in established disease [19]. However, in an era of diminishing erosive damage, the relationship between disease activity and disability may have changed. A longitudinal analysis of the Nijmegen inception cohort actually revealed increasing levels of disability over time [9]. UK data from the Norfolk Arthritis Register (NOAR) revealed similar findings, with increasing levels of reported disability over time, despite reductions in measured disease activity [13]. Of even more relevance is an analysis combining the NOAR cohort with another UK inception cohort that explored HAQ progression over time [16]. Several distinct trajectories were noted; these were predicted by older age, female sex, longer symptom duration, higher DAS28 and lower social class.

Our findings help contextualise the changes in HAQ over time. Whilst we observed an overall reduction in disability, this was possibly driven by a decline in patients 
with severe disease. In contrast, HAQ scores increased within the lower DAS28 groups. This corresponds to the rise in the subjective components of DAS28, which predictably correlate better with patient reported disability. One explanation for these findings is that background levels of disability might be rising, perhaps due to external factors such as the climbing levels of obesity in the general population. However, this is at odds with data from nonRA populations that have reported reductions in disability in the general population [20]. An alternative explanation is that way patients complete HAQ scores may be changing: patients might report disability differently using the HAQ due to higher expectations. The HAQ tool is also limited by floor and ceiling effects. Irrespective of the reason for the changing pattern of HAQ score, it remains a core metric by which effectiveness of RA treatments are judged [21].

Our study has several strengths. We have reported real time data with no exclusion criteria which is likely to be highly representative of RA patients seen in outpatient clinics at the time of each survey. It was also sufficiently large to provide robust assessments of treatment and disease activity. Our study does however have some limitations. The data were collected at consecutive cohorts was cross sectional and did not capture the same patients. As the data collected were anonymized we were uncertain if some patients contributed to more than one cohort. The assessments were carried out by a number of different physicians over the four time periods and there might have been variations in the way individual clinicians assess joint counts. It is also possible that the pattern of follow up in clinics has changed with RA patients who have milder disease being more frequently. Another possibility is that there may have been changes in the level of background disease activity in patients seen in specialist clinics. These are inherent problems in all studies that evaluate temporal changes in disease activity.

\section{Conclusions}

Over the last two decades the management of rheumatoid arthritis has changed. Patients are treated more intensively with both conventional DMARDs and biologics. Disease activity levels have fallen and more patients achieve remission; however, as only a minority of patients are in remission there is still substantial room for improvement. The failure to substantially reduce disability levels is of some concern. It is possible that focusing on reducing disease activity has less impact on disability than was previously considered. It may be particularly important to focus on reducing tender joint counts and patient global assessments, which may be related to non-inflammatory aspects of RA. Finally, further work is needed to understand how to best manage moderate RA, the phenotype that now makes up the largest burden of clinic attenders.

\section{Abbreviations}

DAS28: Disease activity score for 28 joints; DMARD: Disease modifying anti-rheumatic drug; HAQ: Health assessment questionnaire; IQR: Interquartile range; NOAR: Norfolk arthritis register; RA: Rheumatoid arthritis; SD: Standard deviation.

\section{Competing interests}

The authors have no conflicts of interest to declare.

\section{Authors' contributions}

ANM had the main responsibility of writing the paper and overseeing the submission. FI contributed to the data analysis and editing of the paper. ICS, SB, MF, LP were involved in data collection. SS, GHK and JG were involved in the study design and editing of the paper. DLS proposed the idea of the study and contributing to the editing of the paper. All authors have seen and approved the final version of the paper before submission.

\section{Acknowledgements}

The paper presents independent research funded by the National Institute for Health Research (NIHR) as one of its Programme Grants For Applied Research (Grant Reference Number: RP-PG-0610-10066; Programme Title: Treatment Intensities and Targets in Rheumatoid Arthritis Therapy: Integrating Patients' And Clinicians' Views - The TITRATE Programme). The views expressed are those of the authors and not necessarily those of the NHS, the NIHR or the Department of Health.

We also acknowledge support from the NIHR Biomedical Research Centre at Guy's and St Thomas' NHS Foundation Trust in partnership with King's College London.

\section{Author details}

'Department of Rheumatology, King's College Hospital, Denmark Hill, London SE5 9RS, UK. Department of Rheumatology, King's College School of Medicine, King's College London, Weston Education Centre, 10 Cutcombe Road, Denmark Hill, London SE5 9RJ, UK. ³Department of Rheumatology, University Hospital Lewisham, London SE13 6LH, UK.

Received: 13 August 2015 Accepted: 19 January 2016 WPि?

\section{References}

1. Scott DL, Wolfe F, Huizinga TW. Rheumatoid arthritis. Lancet. 2010;25(376): 1094-108.

2. Van Riel PLCM, Schumacher HR. How does one assess early rheumatoid arthritis in daily clinical practice? Best Pract Res Clin Rheumatol. 2001;15:67-76.

3. Bruce B, Fries JF. The stanford health assessment questionnaire: dimensions and practical applications. Health Qual Life Outcomes. 2003;1:20.

4. Atar D, Birkeland Kl, Uhlig T. 'Treat to target': moving targets from hypertension, hyperlipidaemia and diabetes to rheumatoid arthritis. Ann Rheum Dis. 2010;69:629-30.

5. Silman A, Davies P, Currey HLF, Evans SJW. Is rheumatoid artrhitis becoming less severe? J Chronic Dis. 1983;36:891-7.

6. Pincus T, Sokka T, Kautiainen H. Patients seen for standard rheumatoid arthritis care have significantly better articular, radiographic, laboratory, and functional status in 2000 than in 1985. Arthritis Rheum. 2005:52:1009-19.

7. Abelson B, Sokka T, Pincus T. Declines in erythrocyte sedimentation rates in patients with rheumatoid arthritis over the second half of the $20^{\text {th }}$ century. J Rheumatol. 2009;36:1596-9.

8. Finckh A, Choi HK, Wolfe F. Progression of radiographic joint damage in different eras: trends towards milder disease in rheumatoid arthritis are attributable to improved treatment. Ann Rheum Dis. 2006:65:1192-7.

9. Welsing PM, Fransen J, van Riel PL. Is the disease course of rheumatoid arthritis becoming milder? Time trends since 1985 in an inception cohort of early rheumatoid arthritis. Arthritis Rheum. 2005:52:2616-24.

10. Houssien DA, MCKenna SP, Scott DL. The Nottingham health profile as a measure of disease activity and outcome in rheumatoid arthritis. Br J Rheumatol. 1997;36:69-73.

11. Scott DL, Khoshaba B, Choy EH, Kinglsey GH. Limited correlation between the Health Assessment Questionnaire $(\mathrm{HAQ})$ and EuroQol in rheumatoid 
arthritis: questionable validity of deriving quality adjusted life years from HAQ. Ann Rheum Dis. 2007;66:1534-7.

12. Scott IC, Ibrahim F, Johnson D, Scott DL, Kinglsye GH. Current limitations in the management of cardiovascular risk in rheumatoid arthritis. Clin Exp Rheumtol. 2012;30:228-32.

13. Diffin JG, Lunt M, Marshall T, Chipping JR, Symmons DP, Verstappen SM. Has the severity of rheumatoid arthritis at presentation diminished over time? J Rheumatol. 2014;41:1590-9.

14. Kievit W, Fransen J, de Waal Malefijt MC, den Broeder AA, van Riel PL. Treatment changes and improved outcomes in RA: an overview of a large inception cohort from 1989 to 2009. Rheumatology. 2013;52:1500-8.

15. Sokka T, Kautiainen H, Hakkinen A, Hannonen P. Radiographic progression is getting milder in patients with early rheumatoid arthritis. Results of 3 cohorts over 5 years. J Rheumatol. 2004;31:1073-82.

16. Aga $A B$, Lie $E$, Uhlig $T$, et al. Time trends in disease activity, response and remission rates in rheumatoid arthritis during the past decade: results from the NOR-DMARD study 2000-2010. Ann Rheum Dis. 2015;74:381-8.

17. Nikiphorou E, Carpenter L, Morris S, et al. Hand and foot surgery rates in rheumatoid arthritis have declined from 1986 to 2011, but large-joint replacement rates remain unchanged: results from two UK inception cohorts. Arthritis Rheumatol. 2014;66:1081-9.

18. Matcham F, Rayner L, Steer S, Hotopf M. The prevalence of depression in rheumatoid arthritis: a systematic review and meta-analysis. Rheumatology. 2013;52:2136-48.

19. Welsing PM, van Gestel AM, Swinkels HL, Kiemeney LA, van Riel PL. The relationship between disease activity, joint destruction, and functional capacity over the course of rheumatoid arthritis. Arthritis Rheum. 2001;44: 2009-17.

20. Martin LG, Schoeni RF, Freedman VA, Andreski P. Feeling better? Trends in general health status. J Gerontol B Psychol Sci Soc Sci. 2007;62:S11-21.

21. Brennan A, Bansback N, Nixon R, et al. Modelling the cost effectiveness of TNF-alpha antagonists in the management of rheumatoid arthritis: results from the British Society for Rheumatology Biologics Registry. Rheumatology. 2007;46:1345-54

\section{Submit your next manuscript to BioMed Central and we will help you at every step:}

- We accept pre-submission inquiries

- Our selector tool helps you to find the most relevant journal

- We provide round the clock customer support

- Convenient online submission

- Thorough peer review

- Inclusion in PubMed and all major indexing services

- Maximum visibility for your research

Submit your manuscript at www biomedcentral.com/submit

) Biomed Central 